TRANSACTIONS OF THE

AMERICAN MATHEMATICAL SOCIETY

Volume 363, Number 9, September 2011, Pages 4569-4584

S 0002-9947(2011)05477-2

Article electronically published on April 11, 2011

\title{
ON MODULAR FORMS AND THE INVERSE GALOIS PROBLEM
}

\author{
LUIS DIEULEFAIT AND GABOR WIESE
}

\begin{abstract}
In this article new cases of the inverse Galois problem are established. The main result is that for a fixed integer $n$, there is a positive density set of primes $p$ such that $\mathrm{PSL}_{2}\left(\mathbb{F}_{p^{n}}\right)$ occurs as the Galois group of some finite extension of the rational numbers. These groups are obtained as projective images of residual modular Galois representations. Moreover, families of modular forms are constructed such that the images of all their residual Galois representations are as large as a priori possible. Both results essentially use Khare's and Wintenberger's notion of good-dihedral primes. Particular care is taken in order to exclude nontrivial inner twists.
\end{abstract}

\section{INTRODUCTION}

The inverse Galois problem asks whether any given finite group occurs as the Galois group of some Galois extension $K / \mathbb{Q}$.

For any newform $f$ and any prime $\ell$, the projective image $G$ of the $\bmod \ell$ Galois representation attached to $f$ is a Galois group over $\mathbb{Q}$ by Galois theory. If $\ell$ is a socalled nonexceptional prime for $f$, then $G$ is of the type $\mathrm{PSL}_{2}\left(\mathbb{F}_{\ell^{n}}\right)$ or $\mathrm{PGL}_{2}\left(\mathbb{F}_{\ell^{n}}\right)$. If $\ell$ is exceptional, then $G$ is an abelian group, a dihedral group, or $A_{4}, S_{4}$ or $A_{5}$.

In this article we construct families of newforms without exceptional primes (see Theorem 6.2). This is achieved by exploiting the notion of tamely dihedral primes, which are essentially the same as the good-dihedral primes introduced by Khare and Wintenberger in their proof of Serre's modularity conjecture KW1. All the constructed newforms also enjoy the property that they do not have any nontrivial inner twist and are not CM forms (see Section 2).

These techniques, together with some other methods, are applied to the inverse Galois problem for groups of the type $\mathrm{PSL}_{2}\left(\mathbb{F}_{\ell^{n}}\right)$ and $\mathrm{PGL}_{2}\left(\mathbb{F}_{\ell^{n}}\right)$. Our results on that problem fall in two different categories, which we like to call the horizontal direction and the vertical direction. The terminology is explained by Figure 1 If a dot exists at position $(\ell, n)$ in the figure, then it is known that $\mathrm{PSL}_{2}\left(\mathbb{F}_{\ell^{n}}\right)$ is a Galois group over $\mathbb{Q}$. Theorem 1.1 of $\mathrm{Wi}$ is the best result in the vertical direction to this date. It says that in every column (i.e. for every prime $\ell$ ) there

Received by the editors May 26, 2009.

2010 Mathematics Subject Classification. Primary 11F80; Secondary 12F12, 11F11.

Key words and phrases. Modular forms, Galois representations, inverse Galois problem.

The first author was partially supported by the grant MTM2009-07024 from the Ministerio de Ciencia e Innovación (Spain).

The second author acknowledges partial support by the Sonderforschungsbereich Transregio 45 of the Deutsche Forschungsgemeinschaft. Both authors were partially supported by the European Research Training Network Galois Theory and Explicit Methods MRTN-CT-2006-035495.

(C)2011 American Mathematical Society

Reverts to public domain 28 years from publication 


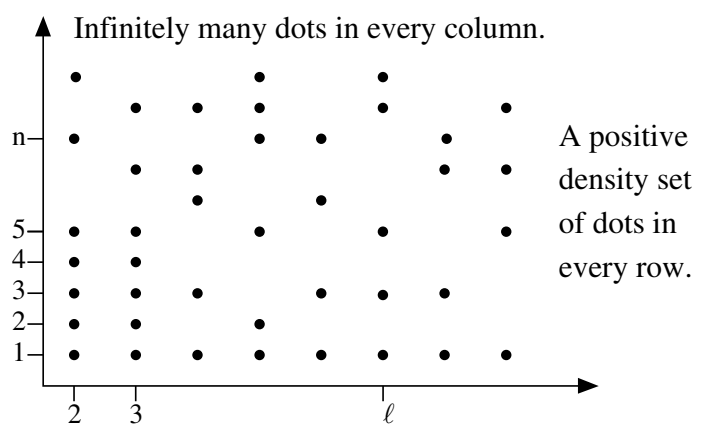

FiguRE 1. Schematic description of the proved cases of $\operatorname{PSL}_{2}\left(\mathbb{F}_{\ell^{n}}\right)$ occuring as a Galois group over $\mathbb{Q}$

are infinitely many dots, i.e. there are infinitely many $n$ such that $\mathrm{PSL}_{2}\left(\mathbb{F}_{\ell^{n}}\right)$ is a Galois group over $\mathbb{Q}$. Except for finitely many columns, this result was reproved in Di2 by different methods. We also prove a similar (but slightly weaker) result in this article (see Remark 6.3), but we do so in a uniform way by using the families without exceptional primes mentioned above. The vertical result has been vastly generalized to many more groups by Khare, Larsen and Savin in [KLS] and [KLS2].

There were several results for small $n$ in the horizontal direction (see [R1, [RV], [DV], Di1]). The main result of this article is that in every row (i.e. for every $n$ ) the set of primes $\ell$ such that $\mathrm{PSL}_{2}\left(\mathbb{F}_{\ell^{n}}\right)$ is a Galois group over $\mathbb{Q}$ has a positive density. More precisely, we prove the following theorem.

Theorem 1.1. Let $n$ be some integer. Then:

(a) There is a positive density set of primes $\ell$ such that $\mathrm{PSL}_{2}\left(\mathbb{F}_{\ell^{n}}\right)$ occurs as the Galois group $\operatorname{Gal}(K / \mathbb{Q})$ for a number field $K$ that ramifies at most at $\ell, \infty$ and two other primes for even $n$ and three other primes for odd $n$.

(b) Assume that $n$ is odd. There is a positive density set of primes $\ell$ such that $\mathrm{PGL}_{2}\left(\mathbb{F}_{\ell^{n}}\right)$ occurs as the Galois group $\operatorname{Gal}(K / \mathbb{Q})$ for a number field $K$ that ramifies at most at $\ell, \infty$ and two other primes.

We finish this introduction by pointing out that our method of obtaining groups of the type $\mathrm{PSL}_{2}\left(\mathbb{F}_{\ell^{n}}\right)$ or $\mathrm{PGL}_{2}\left(\mathbb{F}_{\ell^{n}}\right)$ as Galois groups over $\mathbb{Q}$ via newforms is very general: if a group of this type occurs as the Galois group of a totally imaginary extension of $\mathbb{Q}$, then it is the projective image of the Galois representation attached to a newform by Serre's modularity conjecture, which is now a theorem of KhareWintenberger ([KW1, [KW2]; see also [Ki] and [Di3]).

Proposition 1.2. Let $K / \mathbb{Q}$ be a totally imaginary Galois extension with Galois group $G$ which is either $\mathrm{PSL}_{2}\left(\mathbb{F}_{\ell^{n}}\right)$ or $\mathrm{PGL}_{2}\left(\mathbb{F}_{\ell^{n}}\right)$. Then there exists a modular form $f$ such that its attached projective $\bmod \ell$ Galois representation cuts out the field $K$.

Proof. We interpret the number field $K$ as a projective Galois representation $\bar{\rho}^{\text {proj }}$ : $\operatorname{Gal}(\overline{\mathbb{Q}} / \mathbb{Q}) \rightarrow \mathrm{PGL}_{2}\left(\mathbb{F}_{\ell^{n}}\right)$. Now we lift the representation to a continuous representation $\bar{\rho}: \operatorname{Gal}(\overline{\mathbb{Q}} / \mathbb{Q}) \rightarrow \mathrm{GL}_{2}\left(\overline{\mathbb{F}}_{\ell}\right)$ (this is possible; see e.g. $\mathbb{Q}$ ) and we observe that the lift is necessarily odd. For, the image of complex conjugation in $\mathrm{PGL}_{2}\left(\mathbb{F}_{\ell^{n}}\right)$ 
being nontrivial, it follows that its image under $\bar{\rho}$ is a nonscalar involution. As such, it has determinant -1 . Finally, one invokes Serre's modularity conjecture to obtain the modularity.

Notation. Here we list some notation to be used throughout the article. We denote by $S_{k}(N, \chi)$ the $\mathbb{C}$-vector space of holomorphic cuspidal modular forms of level $N$, weight $k$ and Dirichlet character $\chi$. By $\chi_{\text {triv }}$ we mean the trivial Dirichlet character; instead of $S_{k}\left(N, \chi_{\text {triv }}\right)$ we also write $S_{k}\left(\Gamma_{0}(N)\right)$. If $K$ is a number field, we denote by $\mathcal{O}_{K}$ its ring of integers and by $\mathcal{O}_{K, \Lambda}$ its completion at a maximal ideal $\Lambda \triangleleft \mathcal{O}_{K}$. For a prime $q$, we let $D_{q}$ stand for $\operatorname{Gal}\left(\overline{\mathbb{Q}}_{q} / \mathbb{Q}_{q}\right)$ and denote by $I_{q} \subset D_{q}$ the inertia group. By $W_{q}$ we denote the Weil group of $\mathbb{Q}_{q}$. By $\zeta_{n}$ for an integer $n$ we always denote a primitive $n$-th root of unity.

More notation will be introduced in the text.

\section{Complex multiplicAtion AND inNeR TWists}

In this section we review essential facts on complex multiplication and inner twists. Let $f$ be some cuspidal modular form of level $N$, weight $k$ and Dirichlet character $\chi$ with $q$-expansion $\sum_{n \geq 1} a_{n}(f) q^{n}$ (as usual, $q=q(\tau)=e^{2 \pi i \tau}$ ). The coefficient field of $f$ is defined as $\mathbb{Q}_{f}=\mathbb{Q}\left(a_{n}(f):(n, N)=1\right)$. It has the natural subfield $F_{f}=\mathbb{Q}\left(\frac{a_{n}(f)^{2}}{\chi(n)}:(n, N)=1\right)$, which we call the twist invariant coefficient field of $f$, since it is invariant under replacing the modular form $f$ by any of its twists. The behaviour of the Hecke operators under the Petersson scalar product yields the formula

$$
\overline{a_{p}(f)}=\chi(p)^{-1} a_{p}(f),
$$

whence $\frac{a_{p}(f)^{2}}{\chi(p)}=\left|a_{p}(f)\right|^{2}$. Thus, $F_{f}$ is totally real. It is well known that $\mathbb{Q}_{f}$ is either a CM field or totally real. In particular, if $f$ has trivial nebentype the latter case occurs by equation (2.1).

The modular form $f$ is said to have complex multiplication (CM) if there exists a Dirichlet character $\epsilon$ such that

$$
a_{p}(f \otimes \epsilon)=a_{p}(f) \epsilon(p)=a_{p}(f)
$$

for almost all primes $p$ (i.e. all but finitely many).

A twist of $f$ by a Dirichlet character $\epsilon$ is said to be inner if there exists a field automorphism $\sigma_{\epsilon}: \mathbb{Q}_{f} \rightarrow \mathbb{Q}_{f}$ such that

$$
a_{p}(f \otimes \epsilon)=a_{p}(f) \epsilon(p)=\sigma_{\epsilon}\left(a_{p}(f)\right)
$$

for almost all primes $p$.

For a discussion of inner twists we refer the reader to $[\mathrm{R} 2$ and $[\mathrm{R} 3$. Here we content ourselves by listing some statements that will be needed for the sequel. For the rest of this section we suppose that $f$ does not have CM. Under this assumption $\sigma_{\epsilon}$ is unique (if it exists). Moreover, for two distinct inner twists $\epsilon$ and $\delta$, the field automorphism $\sigma_{\epsilon}$ is different from $\sigma_{\delta}$.

The inner twists and the Dirichlet character of $f$ satisfy the relation

$$
\chi \epsilon^{2}=\sigma_{\epsilon}(\chi) .
$$


If $\chi$ takes only real values (i.e. is trivial or quadratic), it follows that $\epsilon^{2}=1$. This in turn implies

$$
\sigma_{\epsilon}^{2}\left(a_{p}(f)\right)=\sigma_{\epsilon}\left(a_{p}(f) \epsilon(p)\right)=\sigma_{\epsilon}\left(a_{p}(f)\right) \epsilon(p)=a_{p}(f) \epsilon(p)^{2}=a_{p}(f),
$$

whence $\sigma_{\epsilon}^{2}=1$.

The $\sigma_{\epsilon}$ belonging to the inner twists of $f$ form an abelian subgroup $\Gamma$ of the automorphism group of $\mathbb{Q}_{f}$. The field $F_{f}$ is the subfield of $\mathbb{Q}_{f}$ fixed by $\Gamma$. If the nebentype of $f$ only takes real values, it follows that $\Gamma$ is an elementary abelian 2-group and thus that $\left[\mathbb{Q}_{f}: F_{f}\right]$ is a power of 2 .

We define a number field $K_{\Gamma}$ as follows. Consider the inner twists $\epsilon_{1}, \ldots, \epsilon_{r}$ as characters of the absolute Galois group $\operatorname{Gal}(\overline{\mathbb{Q}} / \mathbb{Q})$. Let $K_{\Gamma}$ be the minimal number field on which all $\epsilon_{i}$ for $1 \leq i \leq r$ are trivial, i.e. the field such that its absolute Galois group is the kernel of the map $\operatorname{Gal}(\overline{\mathbb{Q}} / \mathbb{Q}) \stackrel{\epsilon_{1}, \ldots, \epsilon_{r}}{\longrightarrow} \mathbb{C}^{\times} \times \cdots \times \mathbb{C}^{\times}$.

\section{ON THE IMAGES OF MODUlar Galois REPRESEntations}

Let, as before, $f=\sum_{n=1}^{\infty} a_{n} q^{n}$ (with $a_{n} \in \mathbb{C}$ ) be a cuspidal modular form of level $N$, weight $k$ and Dirichlet character $\chi$. We now assume that $f$ is a Hecke eigenform. Also, as before, let $\mathbb{Q}_{f}$ be the coefficient field of $f$; it is naturally a subfield of $\mathbb{C}$. By a construction of Shimura and Deligne and the local Langlands correspondence for $\mathrm{GL}_{2}$ one can attach to $f$ a 2-dimensional compatible system of Galois representations $\left(\rho_{f, \iota}\right)$ of $\operatorname{Gal}(\overline{\mathbb{Q}} / \mathbb{Q})$. We now describe this system, following Khare and Wintenberger [KW0].

The compatible system $\left(\rho_{f, \iota}\right)$ consists of the data of:

(i) for each rational prime $\ell$ and each embedding $\iota: \mathbb{Q}_{f} \hookrightarrow \overline{\mathbb{Q}}_{\ell}$ a continuous semisimple representation $\rho_{f, \iota}: \operatorname{Gal}(\overline{\mathbb{Q}} / \mathbb{Q}) \rightarrow \mathrm{GL}_{2}\left(\overline{\mathbb{Q}}_{\ell}\right)$,

(ii) for each rational prime $q$ a Frobenius semisimple Weil-Deligne representation $r_{q}$ with values in $\mathrm{GL}_{2}\left(\mathbb{Q}_{f}\right)$ such that

(a) $r_{q}$ is unramified for all $q$ outside a finite set,

(b) for each rational prime $\ell$, for each rational prime $q \neq \ell$ and for each $\iota: \mathbb{Q}_{f} \hookrightarrow \overline{\mathbb{Q}}_{\ell}$, the Frobenius semisimple Weil-Deligne representation associated to $\rho_{f, \iota} \mid D_{q}$ is conjugate to $r_{q}$ via the embedding $\iota$,

(iii) a third condition concerning $q=\ell$ (which we do not need).

The system $\rho_{f, \iota}$ is attached to $f$ in the sense that for each rational prime $q \nmid N \ell$, the characteristic polynomial of $\rho_{f, \iota}\left(\operatorname{Frob}_{q}\right)$ is $X^{2}-\iota\left(a_{q}\right) X+\iota\left(\chi(q) q^{k-1}\right)$.

We also introduce a different description of the $\rho_{f, \iota}$, which we will often use below. Let $\iota: \mathbb{Q}_{f} \rightarrow \overline{\mathbb{Q}}_{\ell}$ be an embedding. Denote by $\mathbb{Q}_{f, \iota}$ the closure of $\iota\left(\mathbb{Q}_{f}\right)$ and by $\mathcal{O}_{f, \iota}$ the closure of $\iota\left(\mathcal{O}_{\mathbb{Q}_{f}}\right)$ in $\overline{\mathbb{Q}}_{\ell}$. Let $(\pi)$ be the maximal ideal of the local complete discrete valuation ring $\mathcal{O}_{f, \iota}$. Then $\Lambda:=\mathcal{O}_{\mathbb{Q}_{f}} \cap \iota^{-1}((\pi))$ is a maximal ideal of $\mathcal{O}_{\mathbb{Q}_{f}}$ above $\ell$ and $\mathbb{Q}_{f, \iota}$ can be identified with $\mathbb{Q}_{f, \Lambda}$, the completion of $\mathbb{Q}_{f}$ at $\Lambda$. To simplify notation, we denote by $\mathcal{O}_{f, \Lambda}$ the integers of $\mathbb{Q}_{f, \Lambda}$. Thus, we can identify $\rho_{f, \iota}$ with

$$
\rho_{f, \Lambda}: \operatorname{Gal}(\overline{\mathbb{Q}} / \mathbb{Q}) \rightarrow \mathrm{GL}_{2}\left(\mathcal{O}_{f, \Lambda}\right) .
$$

More precisely, the composition of $\rho_{f, \Lambda}$ with the natural inclusion $\mathcal{O}_{f, \Lambda} \hookrightarrow \overline{\mathbb{Q}}_{\ell}$ equals $\rho_{f, \iota}$.

In this article, we shall mostly be concerned with the reduction of these representations 'modulo $\ell$ ', by which we mean the following. Let $\bar{\rho}_{f, \Lambda}$ be the semisimplification of the reduction of $\rho_{f, \Lambda}$ modulo $\Lambda$ and let $\bar{\rho}_{f, \Lambda}^{\text {proj }}$ be its projective quotient, 
i.e. $\bar{\rho}_{f, \Lambda}$ composed with the natural projection $\mathrm{GL}_{2}\left(\mathbb{F}_{\Lambda}\right) \rightarrow \mathrm{PGL}_{2}\left(\mathbb{F}_{\Lambda}\right)$, where we write $\mathbb{F}_{\Lambda}=\mathcal{O}_{\mathbb{Q}_{f}} / \Lambda$ for the residue field of $\Lambda$.

Theorem 3.1 (Ribet). Suppose that $f$ does not have $C M$ and that $k \geq 2$. Then for almost all maximal ideals $\Lambda$ of $\mathcal{O}_{\mathbb{Q}_{f}}$ (equivalently, for almost all $\iota$ as above), the image $\bar{\rho}_{f, \Lambda}\left(\operatorname{Gal}\left(\overline{\mathbb{Q}} / K_{\Gamma}\right)\right)$ is conjugate to

$$
\left\{g \in \mathrm{GL}_{2}\left(\mathbb{F}_{\lambda}\right): \operatorname{det}(g) \in \mathbb{F}_{\ell}^{\times(k-1)}\right\},
$$

where $K_{\Gamma}$ is the field defined in Section $2, \mathbb{F}_{\ell}$ is the prime field of $\mathbb{F}_{\Lambda}$ and $\mathbb{F}_{\lambda}$ is the residue field of $F_{f}$ at $\lambda=\Lambda \cap F_{f}$, i.e. $\mathbb{F}_{\lambda}=\mathcal{O}_{F_{f}} / \lambda$.

Proof. It suffices to take Ribet [R3, Thm. 3.1] $\bmod \Lambda$.

Corollary 3.2. Under the assumptions of Theorem 3.1, for almost all $\Lambda$ the image $\bar{\rho}_{f, \Lambda}^{\mathrm{proj}}(\mathrm{Gal}(\overline{\mathbb{Q}} / \mathbb{Q}))$ is either $\mathrm{PSL}_{2}\left(\mathbb{F}_{\lambda}\right)$ or $\mathrm{PGL}_{2}\left(\mathbb{F}_{\lambda}\right)$.

Proof. Let $H:=\bar{\rho}_{f, \Lambda}\left(\operatorname{Gal}\left(\overline{\mathbb{Q}} / K_{\Gamma}\right)\right)$ and $G:=\bar{\rho}_{f, \Lambda}(\operatorname{Gal}(\overline{\mathbb{Q}} / \mathbb{Q}))$. As $H$ is a normal subgroup of $G$, it follows that $H /\left(H \cap \overline{\mathbb{F}}_{\ell}^{\times}\right)$is a normal subgroup of $G /\left(G \cap \overline{\mathbb{F}}_{\ell}^{\times}\right)$. By the classification of finite subgroups of $\mathrm{PGL}_{2}\left(\overline{\mathbb{F}}_{\ell}\right)$, it follows for almost all $\Lambda$ that $H$ is either $\mathrm{PSL}_{2}\left(\mathbb{F}_{\lambda}\right)$ or $\mathrm{PGL}_{2}\left(\mathbb{F}_{\lambda}\right)$ and that $G$ is either $\mathrm{PSL}_{2}(\mathbb{F})$ or $\mathrm{PGL}_{2}(\mathbb{F})$ for some extension $\mathbb{F} / \mathbb{F}_{\lambda}$. As $\mathrm{PSL}_{2}(\mathbb{F})$ is simple (for $\# \mathbb{F} \geq 5$ ), $\mathbb{F}=\mathbb{F}_{\lambda}$ follows.

Definition 3.3. Keep the assumptions of Theorem 3.1. A maximal ideal $\Lambda$ of $\mathcal{O}_{\mathbb{Q}_{f}}$ is called exceptional if $\bar{\rho}_{f, \Lambda}^{\text {proj }}(\mathrm{Gal}(\overline{\mathbb{Q}} / \mathbb{Q}))$ is neither $\operatorname{PSL}_{2}\left(\mathbb{F}_{\lambda}\right)$ nor $\mathrm{PGL}_{2}\left(\mathbb{F}_{\lambda}\right)$.

By Corollary 3.2, every form $f$ without CM only has finitely many exceptional primes. The classification of finite subgroups of $\mathrm{PGL}_{2}\left(\overline{\mathbb{F}}_{\ell}\right)$ yields that the group $\bar{\rho}_{f, \Lambda}^{\text {proj }}(\mathrm{Gal}(\overline{\mathbb{Q}} / \mathbb{Q}))$ is either abelian, dihedral, $A_{4}, S_{4}$ or $A_{5}$ for an exceptional prime $\Lambda$.

\section{TAMELY DiHEDRAL REPRESEnTATIONS}

It was observed by Khare and Wintenberger ( KW1] that exceptional primes smaller than a given bound can be avoided by imposing a certain local ramification behaviour of the Galois representation. We shall use this idea in order to exclude $\mathrm{CM}$ and inner twists.

We will formulate the crucial definition in terms of Weil-Deligne representations. As a reference for these, we use $\left[\mathrm{T}\right.$, Section 4 . Let $K$ be a finite extension of $\mathbb{Q}_{q}$ (with $q$ a prime) and $E$ a number field (later $E=\mathbb{Q}_{f}$ ). A 2-dimensional WeilDeligne representation of $K$ with values in $E$ can be described as a pair $(\widetilde{\rho}, \widetilde{N})$ (we use tildes in order to avoid any possible confusion with Galois representations and levels of modular forms), where $\widetilde{\rho}: W_{K} \rightarrow \mathrm{GL}_{2}(E)$ is a continuous representation of the Weil group of $K$ for the discrete topology on $\mathrm{GL}_{2}(E)$ and $\widetilde{N}$ is a nilpotent endomorphism of $E^{2}$ satisfying a certain commutativity relation with $\widetilde{\rho}$.

Definition 4.1. Let $\mathbb{Q}_{q^{2}}$ be the unique unramified degree 2 extension of $\mathbb{Q}_{q}$. Denote by $W_{q}$ and $W_{q^{2}}$ the Weil group of $\mathbb{Q}_{q}$ and $\mathbb{Q}_{q^{2}}$, respectively.

A 2-dimensional Weil-Deligne representation $r_{q}=(\widetilde{\rho}, \widetilde{N})$ of $\mathbb{Q}_{q}$ with values in $E$ is called tamely dihedral of order $n$ if $\widetilde{N}=0$, and there is a tame character $\psi$ : $W_{q^{2}} \rightarrow E^{\times}$whose restriction to the inertia group $I_{q}$ (which is naturally a subgroup of $W_{q^{2}}$ ) is of niveau 2 (i.e. it factors over $\mathbb{F}_{q^{2}}^{\times}$and not over $\mathbb{F}_{q}^{\times}$) and of order $n>2$ such that $\widetilde{\rho} \cong \operatorname{Ind}_{W_{q^{2}}}^{W_{q}}(\psi)$. 
We say that a Hecke eigenform $f$ is tamely dihedral of order $n$ at the prime $q$ if the Weil-Deligne representation $r_{q}$ at $q$ belonging to the compatible system $\left(\rho_{f, \iota}\right)$ is tamely dihedral of order $n$.

If the compatible system $\left(\rho_{f, \iota}\right)$ is tamely dihedral of order $n$ at $q$, then (e.g. by T], 4.2.1) for all $\iota: \mathbb{Q}_{f} \rightarrow \overline{\mathbb{Q}}_{\ell}$ with $\ell \neq q$, the restriction of $\rho_{f, \iota}$ to $D_{q}$ is of the form $\operatorname{Ind}_{\operatorname{Gal}\left(\overline{\mathbb{Q}}_{q} / \mathbb{Q}_{q^{2}}\right)}^{\operatorname{Gal}\left(\overline{\mathbb{A}}_{q} / \mathbb{Q}_{q}\right)}(\iota \%)$. The point in our application is that the reduction modulo $\ell$ is of the very same form, i.e. if $\bar{\psi}_{\Lambda}$ denotes the reduction of $\psi$ modulo $\Lambda$, which is a character of the same order if $\Lambda$ and $n$ are coprime, then

$$
\left.\bar{\rho}_{f, \Lambda}\right|_{D_{q}}=\operatorname{Ind} \underset{\operatorname{Gal}\left(\overline{\mathbb{Q}}_{q} / \mathbb{Q}_{q^{2}}\right)}{\operatorname{Gal}\left(\bar{\psi}_{\Lambda} / \mathbb{Q}_{q}\right)}\left(\bar{\psi}_{\Lambda}\right)
$$

Moreover, if $n=p^{r}$ for some odd prime $p$, then $q \equiv-1 \bmod p$, since the character is of niveau 2. Conversely, if we take $\psi$ to be a totally ramified character of $\operatorname{Gal}\left(\overline{\mathbb{Q}}_{q} / \mathbb{Q}_{q^{2}}\right)$ of order $n$ such that $n$ divides $q+1$ and $(n, q(q-1))=1$, then this automatically ensures that $\psi$ is of niveau 2 .

We also mention that the image of $\widetilde{\rho}$ as in Definition 4.1 is isomorphic as an abstract group to the dihedral group $D_{n}$ of order $2 n$ if $\psi$ is totally ramified and $(n, q(q-1))=1$. This is due to the fact that this condition forces the restriction of the determinant to $\operatorname{Gal}\left(\overline{\mathbb{Q}}_{q} / \mathbb{Q}_{q^{2}}\right)$ to be trivial.

A tamely dihedral prime $q$ is called a good-dihedral prime by Khare and Wintenberger KW1 if some additional properties (of a different nature) are satisfied. Good-dihedral primes play an important role in Khare and Wintenberger's proof of Serre's conjecture.

We now collect some very simple lemmas that will be applied afterwards in order to exclude nontrivial inner twists.

Lemma 4.2. Let $K$ be a topological field, $q$ a prime and $n>2$ an integer coprime to $q(q-1)$. Let $\epsilon: \operatorname{Gal}\left(\overline{\mathbb{Q}}_{q} / \mathbb{Q}_{q}\right) \rightarrow K^{\times}$and $\psi, \psi^{\prime}: \operatorname{Gal}\left(\overline{\mathbb{Q}} / \mathbb{Q}_{q^{2}}\right) \rightarrow K^{\times}$be characters. Suppose that $\psi$ and $\psi^{\prime}$ are both of order $n$.

If $\operatorname{Ind}_{\mathbb{Q}_{q^{2}}}^{\mathbb{Q}_{q}}(\psi) \cong \operatorname{Ind}_{\mathbb{Q}_{q^{2}}}^{\mathbb{Q}_{q}}\left(\psi^{\prime}\right) \otimes \epsilon$, then $\epsilon$ is unramified.

Proof. It directly follows that the order of $\left.\epsilon\right|_{\operatorname{Gal}\left(\overline{\mathbb{Q}}_{q} / \mathbb{Q}_{q^{2}}\right)}$ divides $n$. If $\epsilon$ were ramified, then the order of $\epsilon$ restricted to the inertia group at $q$ would divide $q-1$ times a power of $q$. But this is precisely excluded in the assumption.

Lemma 4.3. Let $K$ be a topological field. Let $q>2$ be a prime, $\epsilon: \operatorname{Gal}\left(\overline{\mathbb{Q}}_{q} / \mathbb{Q}_{q}\right) \rightarrow$ $K^{\times}$a quadratic character and $\rho, \rho^{\prime}: \mathrm{Gal}\left(\overline{\mathbb{Q}}_{q} / \mathbb{Q}_{q}\right) \rightarrow \mathrm{GL}_{2}(K)$ representations such that $\rho^{\prime} \cong \rho \otimes \epsilon$. If $\rho\left(I_{q}\right)$ and $\rho^{\prime}\left(I_{q}\right)$ can be conjugated to lie in the upper triangular matrices such that all elements on the diagonal have odd order, then $\epsilon$ is unramified.

Proof. The oddness of the order of the elements on the diagonal forces the quadratic character $\epsilon$ to be unramified.

Lemma 4.4. Let $K$ be a topological field. Let $q$ be a prime, $\epsilon: \operatorname{Gal}\left(\overline{\mathbb{Q}}_{q} / \mathbb{Q}_{q}\right) \rightarrow K^{\times}$ be a character and $\rho: \operatorname{Gal}\left(\overline{\mathbb{Q}}_{q} / \mathbb{Q}_{q}\right) \rightarrow \mathrm{GL}_{2}(K)$ be a representation. If the conductors of $\rho$ and of $\rho \otimes \epsilon$ both divide $q$, then $\epsilon$ or $\epsilon \operatorname{det}(\rho)$ is unramified.

Proof. By the definition of the conductor, $\rho$ restricted to the inertia group $I_{q}$ is of the form $\left(\begin{array}{cc}1 & * \\ 0 & \delta\end{array}\right)$ with $\delta=\left.\operatorname{det}(\rho)\right|_{I_{q}}$. Consequently, the restriction to $I_{q}$ of $\rho \otimes \epsilon$ looks like $\left(\begin{array}{cc}\epsilon & * \\ 0 & \epsilon \delta\end{array}\right)$. Again by the definition of the conductor, either the restriction of $\epsilon$ to $I_{q}$ or the restriction of $\epsilon \delta$ is trivial. 
Our main result for controlling inner twists and CM is the following theorem.

Theorem 4.5. Let $f \in S_{k}(N, \chi)$ be a normalized Hecke eigenform. Then:

(a) If $\chi$ only takes real values, then any inner twist of $f$ is at most tamely ramified at any odd prime.

(b) Suppose $\chi=\chi_{\text {triv }}$. Let $q \mid N$ be a prime such that for some $\iota: \mathbb{Q}_{f} \rightarrow \overline{\mathbb{Q}}_{\ell}$ with $\ell \neq q$ the image $\rho_{f, \iota}\left(I_{q}\right)$ can be conjugated to lie in the upper triangular matrices such that the diagonal elements have odd order. Then any inner twist of $f$ is unramified at $q$.

(c) Let $q \| N$ be a prime and suppose that $\chi$ is unramified at $q$. Then any inner twist of $f$ is unramified at $q$.

(d) Let $q$ be a prime such that $q^{2} \| N$ and $f$ is tamely dihedral at $q$ of odd order $n \geq 3$ such that $n$ and $q(q-1)$ are coprime. Then any inner twist of $f$ is unramified at $q$.

Proof. (a) If $\chi$ only takes real values, any inner twist is necessarily quadratic (see Section 21), whence it is at most tamely ramified away from 2 .

For (a)-(c) we let $\epsilon$ be an inner twist with corresponding field automorphism $\sigma: \mathbb{Q}_{f} \rightarrow \mathbb{Q}_{f}$. We include the case $\sigma=\mathrm{id}$, when $\epsilon$ comes from CM. For all $\iota: \mathbb{Q}_{f} \rightarrow \overline{\mathbb{Q}}_{\ell}$, we then have

$$
\rho_{f, \iota} \otimes \epsilon \cong \rho_{f, \iota \circ \sigma},
$$

since the traces of any Frobenius element at any unramified prime $p$ are equal:

$$
\operatorname{tr}\left(\left(\rho_{f, \iota} \otimes \epsilon\right)\left(\operatorname{Frob}_{p}\right)\right)=\iota\left(a_{p}(f) \epsilon(p)\right)=\iota\left(\sigma\left(a_{p}(f)\right)\right)=\operatorname{tr}\left(\rho_{f, \iota \circ \sigma}\left(\text { Frob }_{p}\right)\right) .
$$

(b) If $\rho_{f, \iota}\left(I_{q}\right)$ can be conjugated into the upper triangular matrices such that the diagonal elements have odd order, then it follows that the Weil-Deligne representation $r_{q}=(\widetilde{\rho}, \widetilde{N})$ (in the terminology of $\left.[\mathrm{T}], 4.1 .2\right)$ is such that $\widetilde{\rho}$ can also be conjugated into the upper triangular matrices with only odd order elements on the diagonal. Hence, this property also holds for $\rho_{f, \iota \circ \sigma}\left(I_{q}\right)$. Consequently, Lemma 4.3 yields the statement, as $\epsilon$ again has to be at most quadratic in this case.

(c) The conductors at $q$ of both $\rho_{f, \iota}$ and $\rho_{f, \iota \circ \sigma}$ divide $q$. From Lemma 4.4 it hence follows that $\epsilon$ is unramified at $q$, since the determinant of the representation is unramified at $q$.

(d) If $r_{q}$ is tamely dihedral of order $n$ as in the assumption, the restriction of $\rho_{f, \iota}$ to $D_{q}$ is of the form $\operatorname{Ind}_{\operatorname{Gal}\left(\overline{\mathbb{Q}} / \mathbb{Q}_{q^{2}}\right)}^{\operatorname{Gal}\left(\overline{\mathbb{Q}} / \mathbb{Q}_{q}\right)}(\iota \circ \psi)$, and similarly for $\rho_{f, \iota \circ \sigma}$. By Lemma 4.2 $\epsilon$ is unramified at $q$.

Corollary 4.6. Let $f \in S_{k}\left(N, \chi_{\text {triv }}\right)$ be a Hecke eigenform such that for all primes $q \mid N$

- $q \| N$, or

- $q^{2} \| N$ and $f$ is tamely dihedral at $q$ of some order $n \geq 3$ such that ( $n, q(q-$ 1)) $=1$, or

- $\rho_{f, \iota}\left(I_{q}\right)$ can be conjugated into the upper triangular matrices such that all the elements on the diagonal have odd order for some $\iota: \mathbb{Q}_{f} \rightarrow \overline{\mathbb{Q}}_{\ell}$ with $\ell \neq q$.

Then $f$ does not have any nontrivial inner twists and no CM.

Proof. By Theorem 4.5, any inner twist (or character corresponding to CM) is everywhere unramified, and hence trivial. 
The next proposition will be essential in the application of modular forms to the inverse Galois problem in the horizontal direction.

Proposition 4.7. Let $f \in S_{k}\left(N q^{2}, \chi\right)$ be a Hecke eigenform which is tamely dihedral of some order $n>2$ at $q$ such that $(n, q(q-1))=1$. Then $F_{f}$ contains $\mathbb{Q}\left(\zeta_{n}+\overline{\zeta_{n}}\right)$.

Proof. Let $K_{\Gamma}$ be the field defined in Section 2. From Theorem 4.5 it follows that all inner twists are unramified at $q$. Hence, the inertia group $I_{q}$ can be considered as a subgroup of $\operatorname{Gal}\left(\overline{\mathbb{Q}} / K_{\Gamma}\right)$. Let $\Lambda$ be any prime of $\mathcal{O}_{\mathbb{Q}_{f}}$ not dividing $n q$. By assumption, the image $\rho_{f, \Lambda}\left(I_{q}\right)$ contains an element of the form $\left(\begin{array}{cc}\zeta_{n} & * \\ 0 & \overline{\zeta_{n}}\end{array}\right)$. By Theorem 3.1, $F_{f, \lambda}$ contains its trace, i.e. $\zeta_{n}+\overline{\zeta_{n}}$, where $\lambda=\Lambda \cap F_{f}$. This immediately implies that the field extension $F_{f}\left(\zeta_{n}+\overline{\zeta_{n}}\right) / F_{f}$ is of degree 1, as almost all primes are completely split in it.

Alternatively, one could also derive this proposition by similar arguments directly from the Weil-Deligne representation $r_{q}$ at $q$.

\section{Constructions of newforms that are tamely Dihedral AT SOME PRIME}

In this section we collect tools for constructing newforms and consequently Galois representations which are tamely dihedral at some prime.

Theorem 5.1 (Diamond, Taylor: Level Raising). Let $N \in \mathbb{N}, k \geq 2$ and let $p>k+1$ be a prime not dividing $N$. Let $f \in S_{k}(N, \chi)$ be a newform such that $\bar{\rho}_{f, \mathfrak{P}}$ is irreducible with $\mathcal{O}_{\mathbb{Q}_{f}} \triangleright \mathfrak{P} \mid p$. Furthermore, let $q \nmid N$ be a prime such that $q \equiv-1 \bmod p$ and $\operatorname{tr}\left(\bar{\rho}_{f, \mathfrak{P}}\left(\operatorname{Frob}_{q}\right)\right)=0$.

Then there exists a newform $g \in S_{k}\left(N q^{2}, \tilde{\chi}\right)$ such that

(i) $\bar{\rho}_{g, \mathfrak{p}} \cong \bar{\rho}_{f, \mathfrak{P}}$ for some prime $\mathfrak{p} \mid p$ of $\mathcal{O}_{\mathbb{Q}_{g}}$.

(ii) $g$ is tamely dihedral of order $p^{r}$ for some $r>0$ at $q$.

Proof. This is easily deduced from Theorem A of [DT], using the local Langlands correspondence. For details see Corollary 2.6 of Wi.

A very elaborate possibility of prescribing a tamely dihedral prime is Theorem 5.1.4 of [KW1, which does not need the modularity assumption.

To our knowledge, the best result for prescribing the local behaviour at ramified primes for modular Galois representations is the following theorem by Jared Weinstein, which actually holds for Hilbert modular forms. We only formulate it over $\mathbb{Q}$. A global inertial type $\tau$ is a collection of local inertial types $\left(\tau_{\nu}\right)_{\nu}$ (or equivalently - by the local Langlands correspondence - inertial Weil-Deligne parameters) for $\nu$ running through the places of $\mathbb{Q}$. For the precise definitions we refer to We. We just say that the local inertial type at a finite place $\nu$ determines the restriction of any $\Lambda$-adic representation to the inertia group at $\nu$ uniquely for $\mathcal{O}_{\mathbb{Q}_{f}} \triangleright \Lambda \nmid \nu$. Any newform $f$ uniquely determines a global inertial type, which we denote by $\tau(f)$.

Theorem 5.2 (Weinstein). Up to twisting by one-dimensional characters, the set of global inertial types $\tau$ for which there is no newform $f$ with $\tau=\tau(f)$ is finite.

Proof. [We, Corollary 1.2. 
This means that by making the weight large enough, for any global inertial type $\tau$ there is some newform $f$ with $\tau=\tau(f)$. Alternatively, there is always some newform $f$ of a chosen weight with $\tau=\tau(f)$ if enough primes ramify. Weinstein's result is extremely strong and could be used in several places in this article. We, however, chose more classical arguments like level raising.

For the construction of tamely dihedral modular forms via level raising we need the following lemma.

Lemma 5.3. Let $p_{1}, \ldots, p_{r}$ be primes and let $p$ be a prime different from all the $p_{i}$ such that $p \equiv 1 \bmod 4$. Let $\bar{\rho}_{p}^{\operatorname{proj}}: \operatorname{Gal}(\overline{\mathbb{Q}} / \mathbb{Q}) \rightarrow \mathrm{PGL}_{2}\left(\overline{\mathbb{F}}_{p}\right)$ be an odd Galois representation with image $\mathrm{PSL}_{2}\left(\mathbb{F}_{p^{s}}\right)$ or $\mathrm{PGL}_{2}\left(\mathbb{F}_{p^{s}}\right)$ such that the image of any complex conjugation is contained in $\mathrm{PSL}_{2}\left(\mathbb{F}_{p^{s}}\right)$.

The set of primes $q$ such that

(i) $q \equiv p-1 \bmod p^{2}$,

(ii) $q$ splits in $\mathbb{Q}\left(i, \sqrt{p_{1}}, \ldots, \sqrt{p_{r}}\right)$ and

(iii) $\bar{\rho}_{p}^{\operatorname{proj}}\left(\right.$ Frob $\left._{q}\right)$ lies in the same conjugacy class as $\bar{\rho}_{p}^{\operatorname{proj}}(c)$, where $c$ is any complex conjugation,

has a positive density.

Proof. The proof is adapted from KW1, Lemma 8.2, and closely follows the proof of Lemma 3.2 of $\left[\mathrm{Wi}\right.$. Let $L:=\mathbb{Q}\left(\zeta_{p^{2}}, i, \sqrt{p_{1}}, \ldots, \sqrt{p_{r}}\right)$ and let $K / \mathbb{Q}$ be such that $\operatorname{Gal}(\overline{\mathbb{Q}} / K)=\operatorname{ker}\left(\bar{\rho}_{p}^{\text {proj }}\right)$. Conditions (i) and (ii) must be imposed on the field $L$ and condition (iii) on $K$. If $\operatorname{Gal}(K / \mathbb{Q})$ is $\operatorname{PSL}_{2}\left(\mathbb{F}_{p^{s}}\right)$, then the lemma follows directly from Chebotarev's density theorem, as the intersection $L \cap K$ is $\mathbb{Q}$, since $\mathrm{PSL}_{2}\left(\mathbb{F}_{p^{s}}\right)$ is a simple group. If $\operatorname{Gal}(K / \mathbb{Q})$ is $\operatorname{PGL}_{2}\left(\mathbb{F}_{p^{s}}\right)$, the intersection $L \cap K=M$ is either trivial or an extension of $\mathbb{Q}$ of degree 2. By assumption the image of any complex conjugation lies in $\operatorname{Gal}(K / M) \cong \operatorname{PSL}_{2}\left(\mathbb{F}_{p^{s}}\right)$. Hence any $q$ satisfying condition (iii) is split in $M / \mathbb{Q}$. As $p \equiv 1 \bmod 4$, complex conjugation fixes the quadratic subfield of $\mathbb{Q}\left(\zeta_{p^{2}}\right)$, whence any prime $q$ satisfying conditions (i) and (ii) is also split in $M / \mathbb{Q}$. Hence, we may again appeal to Chebotarev's density theorem, proving the lemma.

In the next proposition we show that in certain cases we can 'add' tamely dihedral primes to newforms in such a way that all the local behaviours at the primes dividing the conductor remain essentially the same. The idea behind this proposition is that for a given newform $f$ we choose a newform $g$ such that the compatible systems of Galois representations of $f$ and $g$ are linked $\bmod p$ for a prime $p$ that is large enough to preserve all essential local properties.

Proposition 5.4. Let $f \in S_{k}\left(N, \chi_{\text {triv }}\right)$ be a newform with odd $N$ such that for all $\ell \mid N$

(i) $\ell \| N$ or

(ii) $\ell^{2} \| N$ and $f$ is tamely dihedral at $\ell$ of order $n_{\ell}>2$ or

(iii) $\ell^{2} \mid N$ and $\rho_{f, \iota}\left(D_{\ell}\right)$ can be conjugated to lie in the upper triangular matrices such that the elements on the diagonal all have odd order for some $\iota: \mathbb{Q}_{f} \hookrightarrow$ $\overline{\mathbb{Q}}_{s}$ with a primes $s \neq \ell$.

Let $\left\{p_{1}, \ldots, p_{r}\right\}$ be any finite set of primes.

Then for almost all primes $p \equiv 1 \bmod 4$ there is a set $S$ of primes of positive density which are completely split in $\mathbb{Q}\left(i, \sqrt{p_{1}}, \ldots, \sqrt{p_{r}}\right)$ such that for all $q \in S$ 
there is a newform $g \in S_{k}\left(N q^{2}, \chi_{\text {triv }}\right)$ which is tamely dihedral at $q$ of order $p$ and for all $\ell \mid N$ we have

(ii) $\ell^{2} \| N$ and $g$ is tamely dihedral at $\ell$ of order $n_{\ell}>2$ or

(iii) $\rho_{g, \iota}\left(D_{\ell}\right)$ can be conjugated to lie in the upper triangular matrices such that the elements on the diagonal all have odd order for some $\iota: \mathbb{Q}_{g} \hookrightarrow \overline{\mathbb{Q}}_{s}$ with a prime $s \neq \ell$.

Moreover, $f$ and $g$ do not have any nontrivial inner twists and no CM.

Proof. By Theorem 4.5, $f$ does not have any nontrivial inner twists and no CM. For $p$ we may choose any prime $p \equiv 1 \bmod 4$ which is larger than $N$, larger than $k+1$, coprime to all $n_{\ell}$ and such that there is $\mathfrak{P} \mid p$ with the property that $\bar{\rho}_{f, \mathfrak{P}}$ is irreducible (see Corollary 3.2).

As $S$ we take the set provided by Lemma 5.3 . Note that the assumptions of the lemma are satisfied, as complex conjugation necessarily lies in $\mathrm{PSL}_{2}$, as there are no nontrivial inner twists and -1 is a square in $\mathbb{F}_{p}^{\times}$.

For any $q \in S$, Theorem 5.1 provides us with a newform $g \in S_{k}\left(N q^{2}, \chi\right)$ which is tamely dihedral at $q$ of order $p^{r}>1$. In fact, $r=1$ and $\chi=\chi_{\text {triv }}$. For, as $p^{2}$ does not divide $q^{2}-1$, it follows that there is no niveau 1 or niveau 2 character of the inertia group $I_{q}$ of order $p^{2}$. That $\chi$ is unramified at $q$ is clear, since the determinant of the restriction to inertia at $q$ is $\psi \psi^{q}=\psi \psi^{-1}=1$ (see also the discussion following Definition 4.1). Hence, $\chi$ is a character $(\mathbb{Z} / N \mathbb{Z})^{\times} \rightarrow \mathbb{C}^{\times}$. As there is a prime $\mathcal{O}_{\mathbb{Q}_{g}} \triangleright \mathfrak{p} \mid p$ such that $\bar{\rho}_{f, \mathfrak{P}} \cong \bar{\rho}_{g, \mathfrak{p}}$, the order of $\chi$ is a power of $p$. As $p$ is larger than $N$, it can only be the trivial character.

Next we check that conditions (ii) and (iii) persist for $g$. This follows again from $\bar{\rho}_{f, \mathfrak{P}} \cong \bar{\rho}_{g, \mathfrak{p}}$, since $p$ is 'large enough'. More precisely, we argue as follows. Let $\ell \mid N$ be a prime and denote by $\left(\widetilde{\rho}_{f}, \widetilde{N}_{f}\right)$ the Weil-Deligne representation attached to $f$ at $\ell$.

We now assume that the order of $\widetilde{\rho}_{f}\left(I_{\ell}\right)$ is divisible by an odd prime. Note that this condition is satisfied at all primes $\ell$ in (ii) and (iii). We first claim that $\left.\rho_{f, \mathfrak{P}}\right|_{D_{\ell}}$ is irreducible if and only if $\left.\bar{\rho}_{f, \mathfrak{P}}\right|_{D_{\ell}}$ is irreducible. Since the other direction is trivial, we now assume that $\left.\rho_{f, \mathfrak{P}}\right|_{D_{\ell}}$ is irreducible. This implies that the representation $\widetilde{\rho}_{f}$ of the Weil group of $\mathbb{Q}_{\ell}$ is also irreducible and consequently $\widetilde{N}_{f}=0$. It follows that the projectivization of $\left.\rho_{f, \mathfrak{B}}\right|_{D_{\ell}}$ is $\operatorname{Ind}_{K}^{\mathbb{Q}_{\ell}}(\psi)$ with $K / \mathbb{Q}_{\ell}$ of degree 2 and $\psi$ a nontrivial character of $\operatorname{Gal}\left(\overline{\mathbb{Q}}_{\ell} / K\right)$, which is different from its conjugate by the nontrivial element in $\operatorname{Gal}\left(K / \mathbb{Q}_{\ell}\right)$. Our assumption now means that the order of $\psi$ restricted to $I_{\ell}$ is divisible by an odd prime. Moreover, local class field theory yields that this prime divides $\ell\left(\ell^{2}-1\right)$ and is thus different from $p$, since $p>N$. This implies that the projectivization of $\left.\bar{\rho}_{f, \mathfrak{P}}\right|_{D_{\ell}}$, which is $\operatorname{Ind}_{K}^{\mathbb{Q}_{\ell}}(\bar{\psi}$ ) (with $\bar{\psi}$ the reduction of $\psi$ modulo $\mathfrak{P}$ ), is actually isomorphic to the projectivization of $\left.\rho_{f, \mathfrak{P}}\right|_{D_{\ell}}$. Consequently, $\left.\bar{\rho}_{f, \mathfrak{P}}\right|_{D_{\ell}}$ is irreducible, as claimed.

Now we consider a prime $\ell$ satisfying (ii). In that case we have that $\widetilde{\rho}_{f} \cong$ $\operatorname{Ind}_{W_{\ell^{2}}}^{W_{\ell}}(\psi)$, where $\psi$ is a niveau 2 character of $\operatorname{Gal}\left(\overline{\mathbb{Q}}_{\ell} / \mathbb{Q}_{\ell}\right)$ of order $n_{\ell}$. Hence,

$$
\left.\left.\bar{\rho}_{f, \mathfrak{P}}\right|_{D_{\ell}} \cong \operatorname{Ind}_{\mathbb{Q}_{\ell^{2}}}^{\mathbb{Q}_{\ell}}(\bar{\psi}) \cong \bar{\rho}_{g, \mathfrak{p}}\right|_{D_{\ell}},
$$

with $\bar{\psi}$ the reduction of $\psi$ modulo $\mathfrak{P}$. Consequently, we find for the Weil-Deligne representation $\left(\widetilde{\rho}_{g}, \widetilde{N}_{g}\right)$ of $g$ that $\widetilde{\rho}_{g} \cong \operatorname{Ind}_{W_{\ell^{2}}}^{W_{\ell}}\left(\psi^{\prime}\right)$ and $\widetilde{N}_{g}=0$, where $\psi^{\prime}$ is a character of $\operatorname{Gal}\left(\overline{\mathbb{Q}}_{\ell} / \mathbb{Q}_{\ell^{2}}\right)$ reducing to $\psi$ modulo $\mathfrak{p}$. This means that $\psi^{\prime}=\psi \alpha$ with 
some character $\alpha$ of order a power of $p$. As $p$ does not divide $\ell\left(\ell^{2}-1\right)$, it follows that $\alpha$ is unramified and hence is already a character of $\mathrm{Gal}\left(\overline{\mathbb{Q}}_{\ell} / \mathbb{Q}_{\ell}\right)$. It follows that $\tilde{\rho}_{g} \cong \operatorname{Ind}_{W_{\ell}{ }^{2}}^{W_{\ell}}(\psi) \otimes \alpha$. As, however, $f$ and $g$ both have trivial nebentype and the same weight, the determinant of $\widetilde{\rho}_{g}$ is the same as the determinant of $\widetilde{\rho}_{f}$ and thus $\alpha^{2}=1$, whence $\alpha$ is the trivial character. Consequently, $\widetilde{\rho}_{f} \cong \widetilde{\rho}_{g}$, proving that $g$ is tamely dihedral at $\ell$ of the same order as $f$. We have thus actually proved that the Weil-Deligne representations of $f$ and $g$ at $\ell$ are the same.

Now we consider a prime $\ell \mid N$ in case (iii). As $\left.\rho_{f, \mathfrak{P}}\right|_{D_{\ell}}$ can be conjugated to lie in the upper triangular matrices, the same holds for $\left.\rho_{g, \mathfrak{p}}\right|_{D_{\ell}}$ by the above discussion on the irreducibility. Let $\left(\phi_{1}, \phi_{2}\right)$ and $\left(\psi_{1}, \psi_{2}\right)$ be the characters on the diagonal of $\left.\rho_{f, \mathfrak{P}}\right|_{D_{\ell}}$ and $\left.\rho_{g, \mathfrak{p}}\right|_{D_{\ell}}$, respectively. As under reduction modulo $\mathfrak{P}$ (respectively, $\mathfrak{p}$ ) only $p$-power orders vanish, it follows from the orders of $\phi_{1}$ and $\phi_{2}$ being odd that this is also the case for $\psi_{1}$ and $\psi_{2}$.

That $g$ does not have any nontrivial inner twists and no CM again follows from Theorem 4.5.

Remark 5.5. At two places in the proof of Theorem 1.1 of Wi] it is implicitly used that the newforms $f$ and $g$ do not have any nontrivial inner twists, namely for having $\bar{\rho}_{f, p}(c) \in \mathrm{PSL}_{2}\left(\mathbb{F}_{p^{r}}\right)$ (in Lemma 3.2) and for $\bar{\rho}_{g, l}^{\mathrm{proj}}\left(\mathrm{Frob}_{w}\right) \in \mathrm{PSL}_{2}\left(\mathbb{F}_{l^{t}}\right)$ (the next-to-the last line in the proof of Theorem 1.1). This is easily remedied by excluding inner twists with the help of Proposition 5.4 and a suitable choice of the starting form $f$. If $\ell \notin\{3,5,7,13\}$, we can just take $f \in S_{2}\left(\Gamma_{0}(\ell)\right)$. In the other cases we choose $f$ of level a suitable power of $\ell$ such that $\rho_{f, \iota}\left(D_{\ell}\right)$ can be conjugated into the upper triangular matrices such that the elements on the diagonal all have odd order.

\section{Construction of eigenforms without exceptional primes}

The aim of this section is to construct families of Hecke eigenforms without exceptional primes and without nontrivial inner twists. They also give a uniform way of reproving (a slightly weaker form of) the main result of [Wi], i.e. an application of modular forms to the inverse Galois problem in the vertical direction.

Proposition 6.1. Let $p, q, t$, and $u$ be distinct odd primes and let $N$ be an integer coprime to pqtu. Let $p_{1}, \ldots, p_{m}$ be the prime divisors of $6 N$. Let $f \in S_{2}\left(N q^{2} u^{2}, \chi\right)$ be a Hecke eigenform without CM which is tamely dihedral of order $p^{r}>5$ at $q$ and tamely dihedral of order $t^{s}>5$ at $u$. Assume that $q$ and $u$ are completely split in $\mathbb{Q}\left(i, \sqrt{p_{1}}, \ldots, \sqrt{p_{m}}\right)$ and that $\left(\frac{q}{u}\right)=1$ (and, hence, $\left(\frac{u}{q}\right)=1$ by quadratic reciprocity).

Then $f$ does not have any exceptional primes, i.e. for all maximal ideals $\Lambda$ of $\mathcal{O}_{\mathbb{Q}_{f}}$, the image of $\bar{\rho}_{f, \Lambda}$ is $\mathrm{PSL}_{2}\left(\mathbb{F}_{\lambda}\right)$ or $\mathrm{PGL}_{2}\left(\mathbb{F}_{\lambda}\right)$ in the notation of Theorem 3.1 .

Proof. The argument is similar to that used in Wi], Theorem 1.1, and was inspired by [KW1. Let $\Lambda$ be any maximal ideal of $\mathcal{O}_{\mathbb{Q}_{f}}$, and suppose it lies over the rational prime $\ell$. Due to the tamely dihedral behaviour, $\bar{\rho}_{f, \Lambda}$ is irreducible. For, if $\ell \notin\{p, q\}$, then already $\left.\bar{\rho}_{f, \Lambda}\right|_{D_{q}}$ is irreducible. If $\ell \in\{p, q\}$, then $\ell \notin\{t, u\}$, and already $\left.\bar{\rho}_{f, \Lambda}\right|_{D_{u}}$ is irreducible.

We now suppose that the projective image is a dihedral group, i.e. it is the induction of a character of a quadratic extension $R / \mathbb{Q}$, i.e. $\bar{\rho}_{f, \Lambda}^{\text {proj }} \cong \operatorname{Ind}_{R}^{\mathbb{Q}}(\alpha)$ for 
some character $\alpha$ of $\operatorname{Gal}(\overline{\mathbb{Q}} / R)$. A priori we know from the ramification of $\bar{\rho}_{f, \Lambda}$ that $R \subseteq \mathbb{Q}\left(i, \sqrt{\ell}, \sqrt{q}, \sqrt{u}, \sqrt{p_{1}}, \ldots, \sqrt{p_{m}}\right)$.

Assume first that $\ell \notin\{p, q\}$. In that case we have

$$
\left.\bar{\rho}_{f, \Lambda}^{\mathrm{proj}}\right|_{D_{q}} \cong \operatorname{Ind}_{\mathbb{Q}_{q^{2}}}^{\mathbb{Q}_{q}}(\psi) \cong \operatorname{Ind}_{R_{\mathfrak{Q}}}^{\mathbb{Q}_{q}}(\alpha)
$$

for some prime $\mathcal{O}_{R} \triangleright \mathfrak{Q} \mid q$, where $\psi$ is a niveau 2 character of order $p^{r}$. From this it follows that $q$ is inert in $R$. By assumption, $q$ is split in $\mathbb{Q}\left(i, \sqrt{u}, \sqrt{p_{1}}, \ldots, \sqrt{p_{m}}\right)$, whence $R \in\{\mathbb{Q}(\sqrt{\ell}), \mathbb{Q}(\sqrt{-\ell})\}$ and $\ell \nmid 6 N u$. As $\ell$ is larger than 3 and does not divide the level of $f$ and as the weight is 2 , the field $R$ cannot ramify at $\ell$ either. This was proved by Ribet (see the proof of Proposition 2.2 in [R4]), using results of Raynaud implying that the Serre weight is 2 and thus that the projective image of inertia $I_{\ell}$ is cyclic of order $\ell+1$ or $\ell-1$. We thus obtain a contradiction showing that $\ell \in\{p, q\}$. In particular, $\ell \notin\{t, u\}$. Exchanging the roles $q \leftrightarrow u, p \leftrightarrow t$ and $r \leftrightarrow s$, the very same arguments again lead to a contradiction. Thus, the projective image of $\bar{\rho}_{f, \Lambda}$ is not a dihedral group.

By the classification of the finite subgroups of $\mathrm{PGL}_{2}\left(\overline{\mathbb{F}}_{\ell}\right)$, it remains to exclude $A_{4}, S_{4}$ and $A_{5}$ as projective images. This, however, is clear, since there is an element of order larger than 5 in the projective image.

Theorem 6.2. There exist eigenforms $\left(f_{n}\right)_{n \in \mathbb{N}}$ of weight 2 with trivial nebentype and without nontrivial inner twists and without CM such that

(i) for all $n$ and all maximal ideals $\Lambda_{n} \triangleleft \mathcal{O}_{f_{n}}$, the residual Galois representation $\bar{\rho}_{f_{n}, \Lambda_{n}}$ is nonexceptional and

(ii) for fixed prime $\ell$, the size of the image of $\bar{\rho}_{f_{n}, \Lambda_{n}}$ for $\mathcal{O}_{\mathbb{Q}_{f_{n}}} \triangleright \Lambda_{n} \mid \ell$ is unbounded for running $n$.

Proof. Start with some newform $f \in S_{2}\left(\Gamma_{0}(N)\right)$ for squarefree level $N$. It does not have any nontrivial inner twists and no $\mathrm{CM}$ by Corollary 4.6. Let $p_{1}, \ldots, p_{m}$ be the prime divisors of $6 \mathrm{~N}$.

Let $B>0$ be any bound. Let $p$ be any prime larger than $B$ provided by Proposition 5.4 applied to $f$ and the set $\left\{p_{1}, \ldots, p_{m}\right\}$, so that we get $g \in S_{2}\left(N q^{2}, \chi_{\text {triv }}\right)$ which is tamely dihedral at $q$ of order $p$ and which does not have any nontrivial inner twists and no CM, for some choice of $q$. Now we apply Proposition 5.4 to $g$ and the set $\left\{q, p_{1}, \ldots, p_{m}\right\}$, and we obtain a prime $t>B$ different from $t$ and some $h \in S_{2}\left(N q^{2} u^{2}, \chi_{\text {triv }}\right)$, which is tamely dihedral at $u$ or order $t$ and which is again without nontrivial inner twists and without $\mathrm{CM}$, for some choice of $u$. By Proposition 6.1, the form $h$ does not have any exceptional primes.

We obtain the family $\left(f_{n}\right)_{n \in \mathbb{N}}$ by increasing the bound $B$ step by step, so that elements of larger and larger orders appear in the inertia images.

Remark 6.3. Theorem 6.2 specializes to the following slightly weaker version of Theorem 1.1 of [Wi] concerning the vertical direction of the inverse Galois problem for projective linear groups:

For every prime $\ell$, there is an infinite set of natural numbers $r$ such that $\mathrm{PSL}_{2}\left(\mathbb{F}_{\ell^{r}}\right)$ or $\mathrm{PGL}_{2}\left(\mathbb{F}_{\ell^{r}}\right)$ occurs as a Galois group over $\mathbb{Q}$. 
The new part here is that the same family $\left(f_{n}\right)_{n \in \mathbb{N}}$ can be used for all primes $\ell$. Theorem 1.1 of [Wi] is stronger in the sense that it only concerns $\mathrm{PSL}_{2}$ and that it contains a ramification statement.

Remark 6.4. Note that many choices were made in Theorem 6.2 and that one can imagine many variations in the proof, resulting in many different families.

\section{Applichtion to the inverse Galois problem}

In this section we prove our main result in the horizontal direction, i.e. Theorem 1.1. We make use of the only way known to us to impose some condition on the coefficient field of a newform, namely, by prescribing certain local ramification conditions. They allow us to have a suitable real cyclotomic field inside the twist invariant coefficient field. Such a result is provided by Proposition 4.7, when there exists a tamely dihedral prime. Another such theorem is the following one by Brumer. In Di2 the first author already observed its usefulness in applications to the inverse Galois problem.

Theorem 7.1 (Brumer). Let $f \in S_{2}(N, \chi)$ be a newform without $C M$. If $p^{r_{p}} \| N$, let $s_{p}$ be the least integer larger than or equal to

$$
\frac{r_{p}}{2}-1-\frac{1}{p-1}
$$

Then $\mathbb{Q}\left(\zeta_{p^{s_{p}}}+\overline{\zeta_{p^{s_{p}}}}\right)$ is a subfield of $F_{f}$.

Proof. B, Theorem 5.5 (b), and the introduction.

We could also give a straightforward argument for $r_{p}=3$ along the lines of the proof of Proposition 4.7. The existence of a real cyclotomic field inside the twist invariant coefficient field will allow the application of the following proposition.

Proposition 7.2. Let $F / \mathbb{Q}$ be a finite field extension which contains a cyclic field $K / \mathbb{Q}$ of degree $n$. Then the set of primes $\ell$ such that there is an ideal $\lambda \triangleleft \mathcal{O}_{F}$ dividing $\ell$ of residue degree $n$ has a positive density.

This proposition follows very easily from the following well-known number theoretic statement.

Proposition 7.3. Let $G$ be the Galois group of a normal extension $L / k$ of number fields, $\ell$ be a prime in $\mathcal{O}_{k}$ which is unramified in $\mathcal{O}_{L}$, $\phi$ be any Frobenius automorphism of $\ell$ in $G, H$ be an arbitrary subgroup of $G$, and $F$ be the subfield of $L$ fixed by $H$. Suppose that the right action of the cyclic subgroup $\langle\phi\rangle$ of $G$ partitions the set $H \backslash G$ of right cosets of $H$ into $r$ orbits with $f_{1}, \ldots, f_{r}$ cosets, respectively. Then $\ell$ splits into $r$ primes $\lambda_{i}$ in $\mathcal{O}_{F}$, for which the residual degrees $f\left(\lambda_{i} / \ell\right)$ are given by the numbers $f_{i}$.

Proof. [M, Theorem 33, p. 111].

Proof of Proposition 7.2. Let $L$ be the Galois closure of $F$ over $\mathbb{Q}$ with Galois group $G$. Let $H$ be the subgroup of $G$ such that $F=L^{H}$. By assumption, there is a surjection of groups $G \stackrel{\pi}{\rightarrow} \mathbb{Z} / n \mathbb{Z}$ such that $H$ is contained in $\operatorname{ker}(\pi)$. 
Let $g \in G$ be any element such that $\pi(g)=1 \in \mathbb{Z} / n \mathbb{Z}$ and let $r \geq 1$ be the minimum integer such that $g^{r} \in H$. Then $\left\{H g^{i} \mid i \in \mathbb{N}\right\} \subset H \backslash G$ consists of $r$ elements. Moreover, as $g^{r} \in H \subseteq \operatorname{ker}(\pi)$, it follows that $0=\pi\left(g^{r}\right)=r \in \mathbb{Z} / n \mathbb{Z}$, whence $n \mid r$, say, $r=n m$. Put $\phi=g^{m}$. Then the minimum $s \geq 1$ such that $\phi^{s} \in H$ is equal to $n$. Consequently, the set $\left\{H \phi^{i} \mid i \in \mathbb{N}\right\} \subset H \backslash G$ consists of $n$ elements.

By Chebotarev's density theorem, the set of primes $\ell$ such that the conjugacy class of the Frobenius elements at $\ell$ is in the conjugacy class of $\phi$ in $G$ has a positive density. By Proposition 7.3, every such $\ell$ has the property that there is an ideal $\lambda \triangleleft \mathcal{O}_{F}$ dividing $\ell$ of residue degree $n$, as desired.

Proof of Theorem 1.1. We first prove (a) for even $n$ and (b) for odd $n$ together. Then we prove (a) for odd $n$.

We choose a prime $p$ which is $1 \bmod 2 n$. We also choose an auxiliary prime $N>$ 13 different from $p$. By Proposition 5.4 there exists a prime $q$ (in fact, $q$ can be chosen from a set of primes of positive density) such that there is a newform $f \in S_{2}\left(\Gamma_{0}\left(N q^{2}\right)\right)$ without $\mathrm{CM}$ and without nontrivial inner twists which is tamely dihedral of order $p>1$ at $q$. Further, by Proposition 4.7, the field $F_{f}=\mathbb{Q}_{f}$ contains the maximal totally real subfield of the cyclotomic field $\mathbb{Q}\left(\zeta_{p}\right)$.

As $p \equiv 1 \bmod 2 n$, the number of elements in the group $\operatorname{Gal}\left(\mathbb{Q}\left(\zeta_{p}+\overline{\zeta_{p}}\right) / \mathbb{Q}\right) \cong$ $(\mathbb{Z} / p \mathbb{Z})^{\times} /\{ \pm 1\}$ is divisible by $n$. Hence, there exists a Galois extension $K / \mathbb{Q}$ with Galois group $\mathbb{Z} / n \mathbb{Z}$ such that $K \subseteq F_{f}=\mathbb{Q}_{f}$.

By Proposition 7.2, the set of primes $\ell$ such that there is $\lambda \triangleleft \mathcal{O}_{F_{f}}$ dividing $\ell$ of residue degree $n$ has a positive density. For almost all such $\lambda$, Ribet's large image theorem (Corollary 3.2) now implies that the projective image of the residual Galois representation $\bar{\rho}_{f, \lambda}$ is equal to $\mathrm{PSL}_{2}\left(\mathbb{F}_{\ell^{n}}\right)$ or $\mathrm{PGL}_{2}\left(\mathbb{F}_{\ell^{n}}\right)$.

Assuming that $n$ is even, every determinant (an element of $\mathbb{F}_{\ell}^{\times}$) is a square in $\mathbb{F}_{\ell^{n}}$. Consequently, the projective image is equal to $\mathrm{PSL}_{2}\left(\mathbb{F}_{\ell^{n}}\right)$, proving (a) for even $n$. Assuming, on the other hand, that $n$ is odd, the same reason shows that the projective image is $\mathrm{PGL}_{2}\left(\mathbb{F}_{\ell^{n}}\right)$, proving (b).

Now we prove (a) for odd $n$. We choose a prime $N \equiv 1 \bmod 2 n$. We also choose an auxiliary prime $q_{1} \neq N$ and an auxiliary prime $q_{2} \equiv 3 \bmod 4$ different from $q_{1}$. Let $\chi:\left(\mathbb{Z} / q_{2} \mathbb{Z}\right)^{\times} \rightarrow\{ \pm 1\}$ be the unique odd Dirichlet character. Let $f \in S_{3}\left(q_{1} q_{2} N^{3}, \chi\right)$ be a newform (the space is nonempty, as one can deduce from dimension formulae). The auxiliary prime $q_{1}$ ensures that $f$ does not have CM. For, by the definition of the conductor, the image of inertia $\rho_{f, \Lambda}\left(I_{q_{1}}\right)$ contains (after conjugation) an element of the form $\left(\begin{array}{ll}1 & b \\ 0 & 1\end{array}\right)$ with $b \neq 0$. Moreover, we know that $\left[\mathbb{Q}_{f}: F_{f}\right]$ is a power of 2 , as $\chi$ only takes real values (see Section 2 ).

By Theorem 7.1, the maximal totally real subfield of $\mathbb{Q}\left(\zeta_{N}\right)$ is contained in $F_{f}$. As above, this implies that there is a field $K \subseteq F_{f} \subseteq \mathbb{Q}_{f}$ such that $K / \mathbb{Q}$ is a cyclic extension of order $n$. This time we apply Proposition 7.2 with the field $\mathbb{Q}_{f}$. We get that the set of primes $\ell$ such that there is $\Lambda \triangleleft \mathcal{O}_{\mathbb{Q}_{f}}$ dividing $\ell$ of residue degree $n$ has a positive density. Note that the residual degree of $\lambda=\Lambda \cap F_{f}$ is also equal to $n$ due to the oddness of $n$. For almost all such $\lambda$, Ribet's large image theorem (Corollary 3.2) yields that the image of the residual Galois representation $\bar{\rho}_{f, \Lambda}$ is equal to $\mathrm{PSL}_{2}\left(\mathbb{F}_{\ell^{n}}\right)$ or $\mathrm{PGL}_{2}\left(\mathbb{F}_{\ell^{n}}\right)$.

Note that all determinants are of the form $\pm \mathbb{F}_{\ell}^{\times 2}$. Hence, in order to obtain $\mathrm{PSL}_{2}\left(\mathbb{F}_{\ell^{n}}\right)$ as a projective image, it suffices, and we need to impose $\ell \equiv 1 \bmod 4$. This is possible. For, if $\mathbb{Q}(i)$ is disjoint from the Galois closure of $\mathbb{Q}_{f}$ over $\mathbb{Q}$, the 
condition on $\Lambda$ is independent from $\ell \equiv 1 \bmod 4$. If, however, $\mathbb{Q}(i)$ is contained in the Galois closure of $\mathbb{Q}_{f}$ over $\mathbb{Q}$, then $\mathbb{Q}_{f}$ contains $i$. As $n$ is odd, any $\ell$ such that there is $\Lambda \mid \ell$ of odd residue degree must be $\equiv 1 \bmod 4$.

Remark 7.4. We point out that in the proof of Theorem 1.1 we made many choices. By varying these choices, the density of the set $\ell$ such that $\mathrm{PSL}_{2}\left(\mathbb{F}_{\ell^{n}}\right)$ occurs as a Galois group over $\mathbb{Q}$ will certainly increase. Up to this point, however, we were unable to prove a nontrivial result in this direction.

Moreover, by Weinstein's Theorem, Theorem 5.2, the auxiliary prime $N$ in (a) for even $n$ is not necessary.

\section{REFERENCES}

[B] A. Brumer. The rank of $J_{0}(N)$. Columbia University Number Theory Seminar (New York, 1992). Astérisque No. 228 (1995), 3, 41-68. MR.1330927 (96f:11083)

[DT] F. Diamond, R. Taylor. Non-optimal levels of mod l modular representations. Invent. Math. 115 (1994), 435-462. MR1262939 (95c:11060)

[Di1] L. V. Dieulefait. Newforms, inner twists, and the inverse Galois problem for projective linear groups. J. Th. Nombres Bordeaux 13 (2001), 395-411. MR1879665 (2003c:11053)

[Di2] L. V. Dieulefait. A control theorem for the images of Galois actions on certain infinite families of modular forms, in Modular Forms on Schiermonnikoog, edited by Gerard van der Geer, Ben Moonen and Bas Edixhoven, Cambridge University Press, 2008, 79-84. MR 2530979 (2010j:11083)

[Di3] L. V. Dieulefait. Remarks on Serre's modularity conjecture. Preprint (2006), arXiv: math/ 0603439.

[DV] L. V. Dieulefait, N. Vila. Projective linear groups as Galois groups over $\mathbb{Q}$ via modular representations. J. Symbolic Comput. 30 (2000), 799-810. MR.1800679 (2001k:11093)

[KLS] C. Khare, M. Larsen, G. Savin. Functoriality and the inverse Galois problem. Compos. Math. 144 (2008), no. 3, 541-564. MR.2422339 (2009m:11076)

[KLS2] C. Khare, M. Larsen, G. Savin. Functoriality and the inverse Galois problem II: Groups of type $B_{n}$ and $G_{2}$. Ann. Fac. Sci. Toulouse Math. (6) 19 (2010), no. 1, 37-70. MR2597780

[KW0] C. Khare, J.-P. Wintenberger. On Serre's conjecture for 2-dimensional mod p representations of $\operatorname{Gal}(\overline{\mathbb{Q}} / \mathbb{Q})$, Annals of Mathematics, 169 (2009), 229-253. MR2480604 (2009m:11077)

[KW1] Chandrashekhar Khare, Jean-Pierre Wintenberger. Serre's modularity conjecture. I. Invent. Math. 178 (2009), no. 3, 485-504. MR2551763 (2010k:11087)

[KW2] Chandrashekhar Khare, Jean-Pierre Wintenberger. Serre's modularity conjecture. II. Invent. Math. 178 (2009), no. 3, 505-586. MR2551764 (2010k:11088)

[Ki] Mark Kisin. Modularity of 2-adic Barsotti-Tate representations. Invent. Math. 178 (2009), no. 3, 587-634. MR.2551765(2010k:11089)

[M] D. A. Marcus. Number Fields. Universitext. Springer. MR.0457396 (56:15601)

[Q] J. Quer. Liftings of projective 2-dimensional Galois representations and embedding problems. J. Algebra 171 (1995), no. 2, 541-566. MR1315912 (96b:12009)

[RV] A. Reverter, N. Vila. Some projective linear groups over finite fields as Galois groups over $\mathbb{Q}$. Contemp. Math. 186 (1995), 51-63. MR:1352266 (96g:12006)

[R1] K. A. Ribet, On l-adic representations attached to modular forms. Invent. Math. 28 (1975), 245-275. MR0419358 (54:7379)

[R2] K. A. Ribet, Twists of modular forms and endomorphisms of abelian varieties. Math. Ann. 253 (1980), no. 1, 43-62. MR.594532 (82e:10043)

[R3] K. A. Ribet, On l-adic representations attached to modular forms. II. Glasgow Math. J. 27 (1985), 185-194. MR819838 (88a:11041)

[R4] K. A. Ribet, Images of semistable Galois representations. Olga Taussky-Todd: in memoriam. Pacific J. Math. 1997, Special Issue, 277-297. MR1610883 (99a:11065)

[T] J. Tate, Number Theoretic Background. Proceedings of Symposia in Pure Mathematics. Vol. 33, part 2, 3-26. MR546607 (80m:12009)

[We] J. Weinstein. Hilbert Modular Forms With Prescribed Ramification. Int. Math. Res. Not. IMRN, 2009, no. 8, 1388-1420. MR2496768 (2010f:11070) 
[Wi] G. Wiese. On projective linear groups over finite fields as Galois groups over the rational numbers in Modular Forms on Schiermonnikoog, edited by Gerard van der Geer, Ben Moonen and Bas Edixhoven, Cambridge University Press, 2008, 343-350. MR 2530980 (2010i:11079)

Departament d’Àlgebra i Geometria, Facultat de Matemàtiques, Universitat de Barcelona, Gran Via de les Corts Catalanes, 585, 08007 Barcelona, Spain

E-mail address: ldieulefait@ub.edu

Institut für Experimentelle Mathematik, Universität Duisburg-Essen, Ellernstrasse 29, 45326 Essen, Germany

E-mail address: gabor.wiese@uni-due.de

$U R L:$ http://maths.pratum.net 